\title{
MEASUREMENTS OF VORTEX GENERATION AND MOTION AT WIND WAVE SURFACES
}

\author{
Kohsei Takehara ${ }^{1}$, T. Goji Etoh ${ }^{2}$, Yasuhide Takano ${ }^{3}$ and Gozo Tsujimoto ${ }^{4}$
}

\begin{abstract}
Vortices generated at water surface under wind wave condition are most important phenomena for mass exchange between atmosphere and ocean. To extract vortices from randomly distributed velocity vectors obtained by a PTV, a new method to accurately estimate vorticity is proposed. The proposed method uses the Moving Least Square method which has been developed for grid-less numerical simulation. The optimal size of fitting area is derived theoretically and is confirmed by Monte Carlo simulation. Comparison of the accuracy between the proposed method and the commonly used method shows the advantage of the proposed method. The developed method is applied to the measurements of vorticity near the wind wave interfaces. The results show the generation and movements of the vortices at water surfaces.
\end{abstract}

Keywords: wind waves; Vortex Motion; Particle Tracking Velocimetry; Moving Least Square Method

\section{INTRODUCTION}

Turbulent structures at air-water interfaces under wind wave conditions are most important factor that enhances gas transfer between the atmosphere and the ocean. Especially, vortex motions near the air-sea interfaces greatly influence the gas transfer at sir-water interfaces (Dean et. al. 2002). However, it is difficult to measure the velocity fields near the water surface by the standard fixed-point velocimetry, such as laser Doppler velocimetry or hot film velocimetry, because of the movements of water surfaces (Komori et. al., 1993). Therefore, the gas transfer rate at the air-water surfaces has been related to the turbulent properties obtained away from the air-water surface.

To understand the turbulent structure near the wind wave surface, Toba et al. (1975) observed flow pattern near the surface by using flow visualization techniques. Their results show some significant properties of wind wave turbulence. However, due to the strong turbulence near the wind wave surfaces where the tracers mixed up quickly, it was very difficult to visualize flow fields sufficiently in their experiments.

In the last few decades, a planar flow measurement technique has been developed by combining flow visualization techniques and image processing techniques, called the Image Velocimetry, IV. The most well-known IV is Particle Image Velocimetry, PIV (Adrian, 1990). The PIV measures velocity vectors by analyzing movements of tracer particles between the sequential images which are captured by a digital video camera. The advantages of the PIV are listed as follows,

1) The PIV can measure instantaneous velocity distributions on a two dimensional plane. Time sequential velocity distributions can be obtained using a video camera. When more than two cameras are used for capturing the particle images, the PIV can measure three dimensional velocity distributions.

2) The PIV enables measurement of a velocity distribution near a moving boundary like a wind wave surface.

For these reasons, some researchers have applied the PIV to the measurements of wind wave fields (Banner and Peirson, 1998, Siddiqui et al., 2001, Takehara et al. 2002, 2004).

The authors developed a Particle Tracking velocimetry, PTV, which is one of the PIV and is tracking individual tracer particles automatically. The developed PTV consists of Kalman filter and chi-square test (Etoh and Takehara, 1995, Takehara, et al., 2000). The PTV was also applied to measurements of some flow fields and the results show its validity of the proposed PTV (e.g. Takehara et al. 2003, Devasenathipathy, et al., 2003, Ootsuka et al., 2003).

Velocity vectors obtained by PTV distributed randomly in space because velocity vectors are measured at positions of tracer particles. When the vorticity is estimated from a velocity distribution measured by a PTV, the velocity distributions are usually converted to velocities on a regular grid. In

\footnotetext{
${ }^{1}$ Department of Civil and Environmental Engineering, Kinki University, 3-4-1 Kowakae, Higashi-Osaka, Osaka, 5778502, Japan

${ }^{2}$ The same as the above

${ }^{3}$ The same as the above

${ }^{4}$ Department of Civil Engineering, Kobe City College of Technology,8-3 Gakuen-Higashi, Nishi-Ku, Kobe, Hyogo, 651-2194, Japan
} 


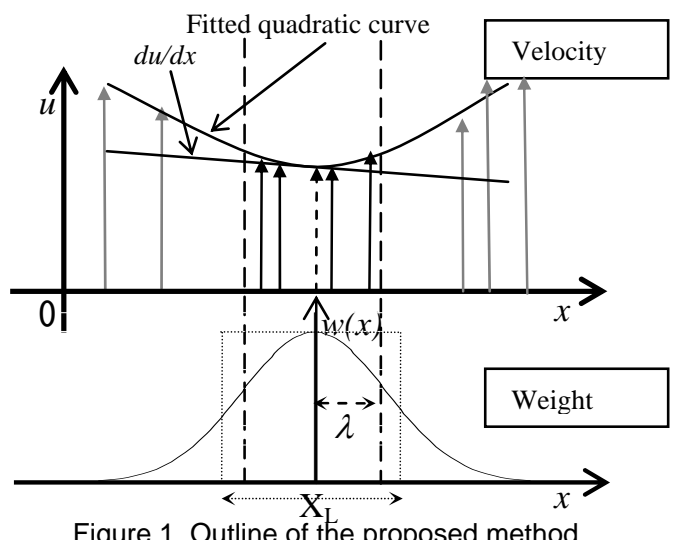

this case, the estimated vorticities include not only the errors generated in the deferential scheme for estimation of voriticies, but also the errors generated in the conversion from the randomly distributed vectors to the grid vectors.

In this paper, we propose a new method to estimate accurate vorticity directly from randomly distributed velocities obtained by PTV. The proposed method has used the Moving Least Square method, what is called the MLS method, which is a grid-less scheme used in numerical simulations. In the MLS method, physical quantities, such as velocities, pressure and concentrations, and their differential coefficients are estimated by a curve fitting method from randomly distributed physical quantities. When the curve fitting method is applied to the estimation of vorticities for randomly distributed velocity vectors, a certain fitting area has to be set on the two dimensional velocity distributions. In this research, a two dimensional quadratic surface is fitted to the velocity vectors inside the fitting area by the least square method. If the fitting area is too small, the number of velocity vectors inside the fitting area decrease. In this case, the results of the MLS are strongly affected by the errors included in the velocity vectors obtained by the PTV. Conversely, if the fitting area is too large, the results of the MLS are affected by the truncation error caused by the fitting quadratic surface. This fact indicates that an optimal size of the fitting area exists for the MLS. The optimal size of the fitting area for the proposed MLS is derived theoretically and the coefficients of the theoretical equation are determined by a numerical simulation in this paper. The accuracy of the proposed method is compared with that of the commonly used method. The proposed method is applied to the vorticity measurements under wind wave conditions. The authors have successfully obtained measurement data for generation of vortices at the wind wave surfaces as well as for downward movement of the generated vortices.

\section{MOVING LEAST SQUARE METHOD (MLS)}

The Moving Least Square method is one of the grid-less scheme for the numerical simulation of fluid flow (Yagawa and Sakai, 1997). In the MLS method, a polynomial surface is fitted to physical quantity, such as velocity, pressure, density, etc., in a certain area. The least square method is applied to the surface fitting in the MLS method. The differential coefficients of the physical quantities are derived from the differentiation of the fitted polynomial function. The MLS method can estimate the physical quantities and their differential coefficients from the surrounding data. In this paper, the MLS method is applied to directly estimate vorticities from randomly distributed velocity vectors which are measured by a PTV.

In this research, the following quadratic surfaces are fitted in a certain area.

$$
\begin{aligned}
& u(x, y)=a_{u} x^{2}+b_{u} x y+c_{u} y^{2}+d_{u} x+e_{u} y+f_{u} \\
& v(x, y)=a_{v} x^{2}+b_{v} x y+c_{v} y^{2}+d_{v} x+e_{v} y+f_{v}
\end{aligned}
$$

where $\left(a_{u}, b_{u}, c_{u}, d_{u}, e_{u}, f_{u}\right)$ and $\left(a_{v}, b_{v}, c_{v}, d_{v}, e_{v}, f_{v}\right)$ are coefficients of the fitted quadratic surfaces. The coefficients are determined by a least square method with measured randomly distributed velocity vectors. The vorticity at the location $(x, y)$ is derived from the fitted quadratic surfaces as follows,

$$
\omega=\frac{\partial v}{\partial x}-\frac{\partial u}{\partial y}=\left(2 a_{v} x+b_{v} y+d_{v}\right)-\left(2 c_{u} y+b_{u} x+e_{u}\right)
$$




\section{OPTIMAL SIZE OF FITTING AREA OF THE PROPOSED MLS METHOD}

There are two kinds of errors related to the MLS method. One is the random error and the other is the shift error. The random error is caused by the measurement error which always occurs when the velocity are measured. In this research, the measurement errors are mainly occurred in the image processing of finding centroids of tracer particles on an image plane. In the MLS method, the estimation error by the fitting surface in a certain area is affected by the measurement errors. The random error strongly depends on the number of measured velocities in the fitting area. On the other hand, the shift error is caused by the truncation error of fitting quadratic surface in MLS. The truncation error depends on the size of the fitting area.

There is a trade off relationship between the random error and the shift error. When the size of the fitting area is set to too small to reduce the shift error, random error becomes large because of the insufficient number of measured velocities. When the size of the fitting area is set to large to reduce the random error, the shift error becomes large because of the truncation error. This fact indicates that the optimal size of the fitting area in the MLS exists.

In this research, the Gaussian weighted least square method is applied to the fitting quadratic surface in the MLS. For simplicity, one dimensional form is used for the following explanation.

$$
W(x)=\frac{1}{\sqrt{2 \pi} \lambda} \exp \left\{-\frac{\left(x-x_{m}\right)^{2}}{2 \lambda^{2}}\right\}
$$

The size of fitting area in the MLS is determined by the deviation, $\lambda$, of the Gaussian weight function. The equivalent width, $X_{L}$, of the above Gaussian distribution becomes

$$
X_{L}=\sqrt{2 \pi} \lambda \cong 2.507 \lambda \text {. }
$$

The optimal size of fitting area, $\lambda_{\text {opt }}$, is already derived by Etoh et. al. (1999). Following derivation of the theoretical optimal size of fitting area is based on the paper.

At first, we focus on the shift error. The function of the fitting curve is expanded around $x_{m}$ by Taylor expansion.

$$
y=f(x)=\delta_{0}+\frac{\delta_{1}}{1 !}\left(x-x_{m}\right)+\frac{\delta_{2}}{2 !}\left(x-x_{m}\right)^{2}+\frac{\delta_{3}}{3 !}\left(x-x_{m}\right)^{3}+\frac{\delta_{4}}{4 !}\left(x-x_{m}\right)^{4}+\varepsilon_{x}
$$

The $\delta_{n}$ is the n-th order differential coefficient, and $\varepsilon_{x}$ is the sum of higher order term than the 4-th order term. The first order differential coefficient is approximated by the central difference as follows,

$$
\frac{d f}{d x} \cong \frac{f\left(x_{m}+\Delta x\right)-f\left(x_{m}-\Delta x\right)}{2 \Delta x}=\delta_{1}+\frac{\delta_{3}}{3 !} \Delta x^{2}+\frac{\varepsilon_{x_{m}+\Delta x}-\varepsilon_{x_{m}}-\Delta x}{2 \Delta x} .
$$

Because the $\delta_{1}$ equals to $d f / d x$, the third order term becomes main error component for the surface fitting in the MLS. From this result, the shift error, $\varepsilon_{s}$, of the MLS is expressed as follows.

$$
\varepsilon_{s} \sim \frac{\delta_{3}}{6} \Delta x^{2} .
$$

The $\Delta x$ can be related to a half of the equivalent width, $X_{L}$.

$$
\varepsilon_{s} \sim \frac{\delta_{3}}{6}\left(\frac{X_{L}}{2}\right)^{2} \sim \delta_{3} \lambda^{2} .
$$

Finally, the shift error is expressed as follow,

$$
\varepsilon_{s}=\beta_{s} \delta_{3} \lambda^{2},
$$

where the $\beta_{\mathrm{s}}$ is a dimensionless coefficient.

Next, we focus on the random error. The random error is inversely proportional to the square root of the number, $n$, of the measured velocities in the fitting area, and proportional to magnitude of random errors, $\sigma_{\varepsilon}$. Therefore, the random error, $\varepsilon_{N}$, becomes as follows,

$$
\varepsilon_{N} \sim \sigma_{\varepsilon} n^{-1 / 2} X_{L}^{-1} \text {. }
$$

The tracer particle density per unit area, $\rho$ (particle $/$ pixel $^{2}$ ), is defined as follows,

$$
n=\rho X_{L}^{2}=\rho \gamma \lambda^{2} \text {. }
$$

Finally, the random error, $\varepsilon_{N}$, is expressed as follows,

$$
\varepsilon_{N}=\beta_{N} \sigma_{\varepsilon} \rho^{-1 / 2} \lambda^{-2} \text {. }
$$

Square of the total error, $\varepsilon_{T}$, in the MLS method is equal to the sum of squared random error and squared shift error, because the random error and the shift error are independent each other. 


$$
\varepsilon_{T}^{2}=\varepsilon_{S}^{2}+\varepsilon_{N}^{2}=\beta_{S}^{2} \delta_{3}^{2} \lambda^{4}+\beta_{N}^{2} \sigma_{\varepsilon}^{2} \rho^{-1} \lambda^{-4}
$$

Base on the least square method, the optimal fitting size, $\lambda_{\text {opt }}$, of the MLS method is derived from the Eq. 13.

$$
\begin{aligned}
& \frac{\partial \varepsilon_{T}^{2}}{\partial \lambda}=4 \beta_{S}^{2} \delta_{3}^{2} \lambda_{\text {opt }}^{3}-4 \beta_{N}^{2} \sigma_{\varepsilon}^{2} \rho^{-1} \lambda_{\text {opt }}^{-5}=0 \\
& \lambda_{\text {opt }}=\beta_{T} \delta_{3}^{-1 / 4} \sigma_{\varepsilon}^{1 / 4} \rho^{-1 / 8}
\end{aligned}
$$

Here, $\beta_{T}$ is the coefficient which consists of $\beta_{S}$ and $\beta_{N}$.

\section{MONTE CARLO SIMULATION FOR THE OPTIMAL SIZE OF THE FITTING AREA}

The Monte Carlo simulation is used to determine the coefficient, $\beta_{T}$, of the Eq. 14 . The tracer particles are randomly distributed on an image of size 1024 by 1024 pixels. The number of the tracer particles is changed from 500 to 10,000 . The movement of the tracer particles during $\Delta t$ is given by the following equations.

$$
\begin{aligned}
& \Delta \xi(x, y)=A \sin (2 \pi x / L) \sin (2 \pi y / L) \\
& \Delta \psi(x, y)=A \cos (2 \pi x / L) \cos (2 \pi y / L)
\end{aligned}
$$

where, $\mathrm{L} / 2$ is the size of vortex and $\mathrm{A}$ is the maximum displacement of tracer particles during $\Delta t$. In this simulation, $\mathrm{L} / 2$ and $A$ are fixed as 512 pixel and 10 pixel, respectively.

The vorticity is defined as

$$
\omega=\frac{\partial v}{\partial x}-\frac{\partial u}{\partial y}=\left(\frac{\partial(\Delta \psi)}{\partial x}-\frac{\partial(\Delta \xi)}{\partial y}\right) / \Delta t=\underline{\omega} / \Delta t .
$$

Here, $\underline{\omega}$ is a dimensionless vorticity.

In order to investigate the dependency of the random error on the accuracy of the proposed method, the pseudo-random white Gaussian noises are added to the displacement, $\Delta \xi$ and $\Delta \psi$. The expectation of the white Gaussian noise is fixed to 0 , and the standard deviation, $\mathrm{S}_{\mathrm{r}}$, of the white Gaussian noise is changed at $0.25,0.5,1.0,2.0$ and $5.0(\mathrm{pixel} / \Delta \mathrm{t})$. For each condition of the simulation, ten image data are generated. The proposed vorticity estimation method is applied for the simulation data.

For the investigation of the dependence of the third order differential coefficient on the accuracy of the proposed method, the size of the vortex, L/2, is changed between 128, 256 and 512 pixels. The maximum displacement, $\mathrm{A}$, the number of tracer particles, $\rho$, and the standard deviation of white Gaussian noise, $\mathrm{S}_{\mathrm{r}}$, are fixed at 10 pixel, 5000, and 1.0 pixel, respectively. The size of fitting area of the MLS method is changed from 7.07 to 106.07 pixels with the interval of 7.07 pixels. The optimal size of the fitting area, $\lambda_{\text {opt }}$, is defined by the size at which the estimation error becomes minimum. The minimum estimation error is determined as the minimum point of the fitting quadratic curve by using three data points of which centre is the smallest estimation error. Vorticities are estimated by the

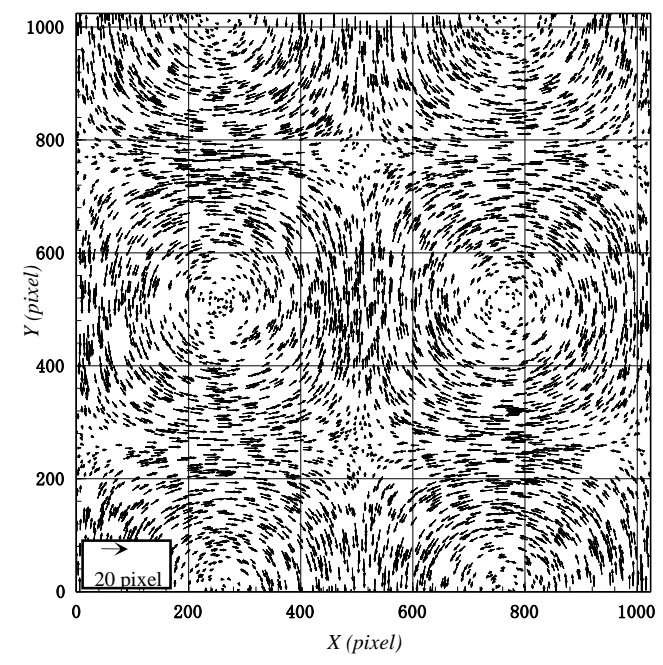

Figure 2. Flow field used in the Simulation (Particle number 5,000) 


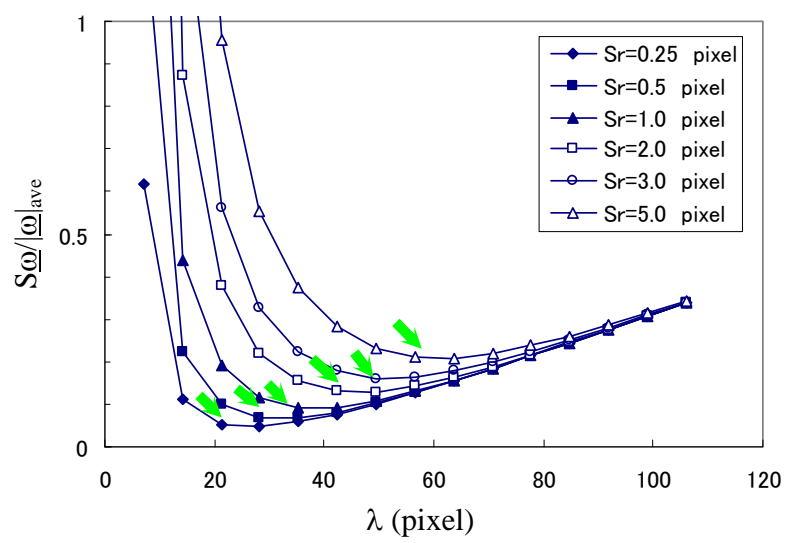

Figure 3. Examples of relation between fitting size, $\lambda$, and relative error (particle number 5,000 , Arrows indicated the smallest relative error for constant random errors)

proposed method on a regular grid of which size is 30 pixels. Fig. 2 shows an example of the velocity distribution used in this research. The number of particle is 5000 without any random noise.

\section{RESULTS AND DISCUSSION}

Fig. 3 shows the examples of the dependency of the estimation error on the fitting area size. The horizontal axis indicates the fitting area size, and the vertical axis indicates relative standard deviation of estimation error, $S_{|\underline{\omega}|}$, normalized by the absolute average of vorticity in one vortex cell, $|\underline{\omega}|_{\text {ave }}$. The results from the Fig. 3 are as follows.

1) When the size of fitting area becomes smaller, the estimation error increases rapidly due to the random noise.

2) When the size of fitting area becomes larger, the estimation error increases gradually by the shift error.

3) When the random error, $S_{r}$, becomes larger, the smallest estimation error increases.

4) For any constant random error, there is an optimal size which has the smallest estimation error. The smallest errors for the constant are indicated as arrows in Fig. 3.

The dependency of the size of optimal fitting area, $\lambda_{\text {opt }}$, on the particle density, $\rho$ (number / $\mathrm{pixel}^{2}$ ), is shown in Fig. 4. The results from the Fig. 4 are as follows.

1) When the random error of the white Gaussian noise increases, the optimal fitting size, $\lambda_{\text {opt }}$, increases.

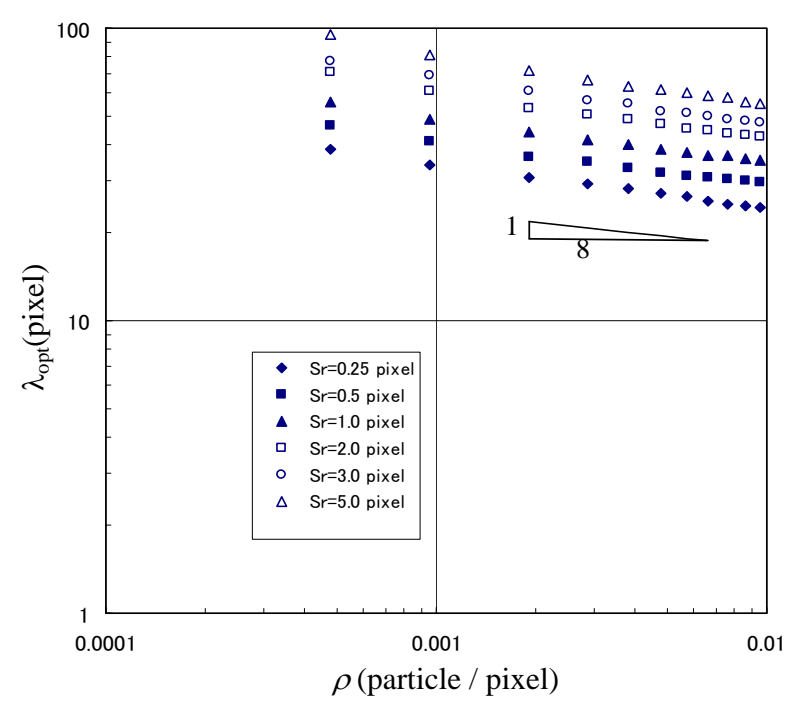

Figure 4. Particle density, $\rho$, vs. optimal fitting size, $\lambda_{\text {opt }}$ 


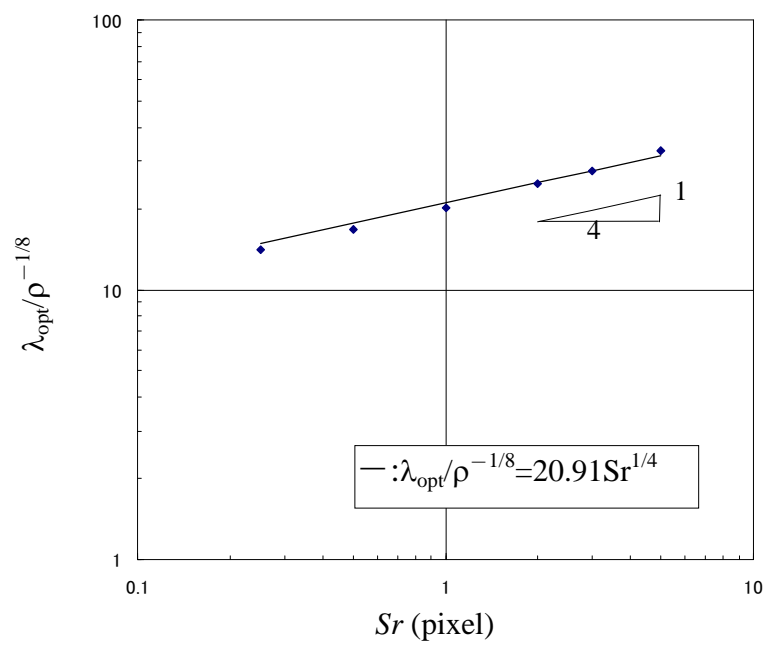

Figure 5. Random error, $S_{r}$, vs. optimal fitting size, $\lambda_{\text {opt }}$

2) For a constant random error, $S_{\mathrm{r}}$, the optimal fitting size, $\lambda_{\text {opt }}$, decreases with the increase of the particle density, $\rho$.

3) The optimal fitting size, $\lambda_{\text {opt }}$, is proportional to the particle density, $\rho$, to the $-1 / 8$ power. This result corresponds exactly to Eq. 14.

The relationship between the optimal fitting size, $\lambda_{\text {opt }}$, and the random error, $S_{r}$, is shown in Fig. 5 . From the results of Fig. 4, we assume the following relation.

$$
\lambda_{\text {opt }}=\alpha \rho^{-1 / 8}
$$

Here, the $\alpha$ is a coefficient for a constant random error, $S_{\mathrm{r}}$. The coefficient, $\alpha$, is calculated for each random error. The horizontal axis indicates the random error, $\mathrm{S}_{\mathrm{r}}$, and the vertical axis indicates the coefficient of Eq.17, $\alpha$. The results from the Fig. 5 are as follows.

1) The optimal fitting area size, $\lambda_{\text {opt }}$, is proportional to the random error to the $1 / 4$ power. This result corresponds exactly to Eq. 14 .

2) The relationship between the coefficient, $\alpha$, and the random error, $S_{r}$, is derived by the least square method with results from the simulation. The relationship is as follows.

$$
\lambda_{\text {opt }} / \rho^{-1 / 8}=20.91 \cdot S_{r}{ }^{1 / 4}
$$

The dependency of the optimal fitting size, $\lambda_{\text {opt }}$ on the third order differential coefficient of velocity distribution function, $\delta_{3}$, is shown in Fig. 6 . As a result, the optimal fitting area size is proportional to the third order differential coefficient, $\delta_{3}$, to the $-1 / 4$ power, which corresponding exactly to Eq. 14.

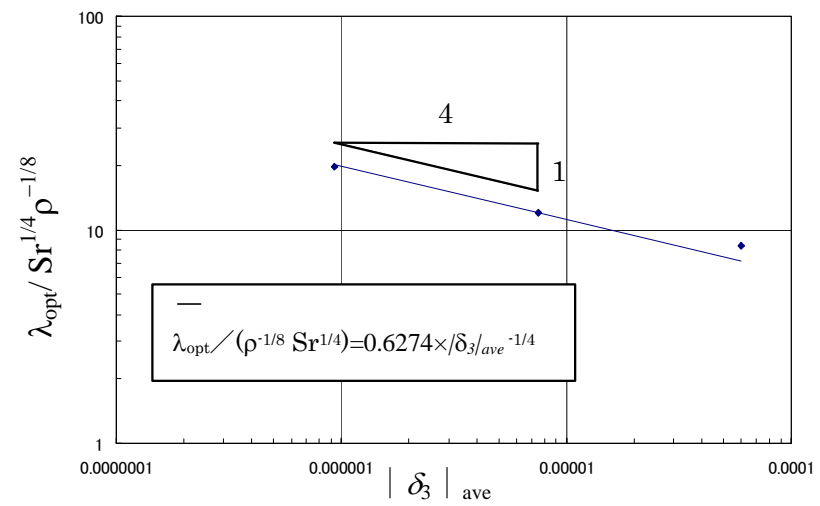

Figure 6. Third order differential coefficient $\left|\delta_{3}\right|$ vs. optimal fitting size, $\lambda_{\text {opt }}$ 


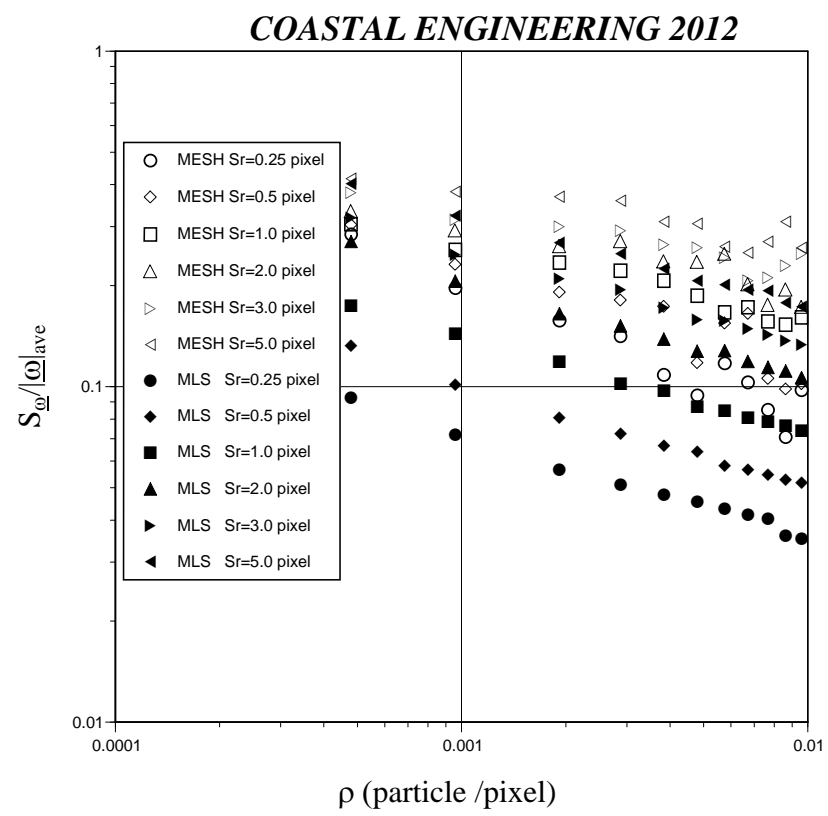

Figure 7. Comparison of the accuracy of the proposed method with that of the commonly used method

Finally, we get the optimal fitting size, $\lambda_{\text {opt }}$ in the proposed method from the Eq. 14 as follows.

$$
\begin{aligned}
& \lambda_{\text {opt }}=\beta_{T} \cdot\left|\delta_{3}\right|^{-1 / 4} \cdot \rho^{-1 / 8} \cdot S_{r}^{1 / 4} \\
& \beta_{T}=0.6274
\end{aligned}
$$

Next a comparison of the accuracy of the proposed method with a commonly used method is carried out. As the commonly used method of vorticity estimation for PTV results, the combination of grid conversion method from randomly distributed velocity data to grid data and the circulation method for estimation of vorticity on the grid data are applied to the same simulation data. Fig. 7 shows the results of the comparison of the accuracies of the two methods. The open symbols indicate the commonly used method and the solid symbols indicate the proposed method. From the Fig. 10, the proposed method is more accurate than the commonly used method in all cases.

The reason why the proposed method is more accurate than the commonly used method is as follows.

1) The proposed method can estimate vorticity from randomly distributed velocity vectors directly.

2) The theoretical optimal fitting area size is derived.

\section{Experimental method and apparatus}

In the experiment, the wind wave tank installed in the hydraulic laboratory of Kinki University was used. The wind wave tank has a length of $16 \mathrm{~m}$, a height of $0.5 \mathrm{~m}$, and a width of $0.5 \mathrm{~m}$. Fig. 8 is a

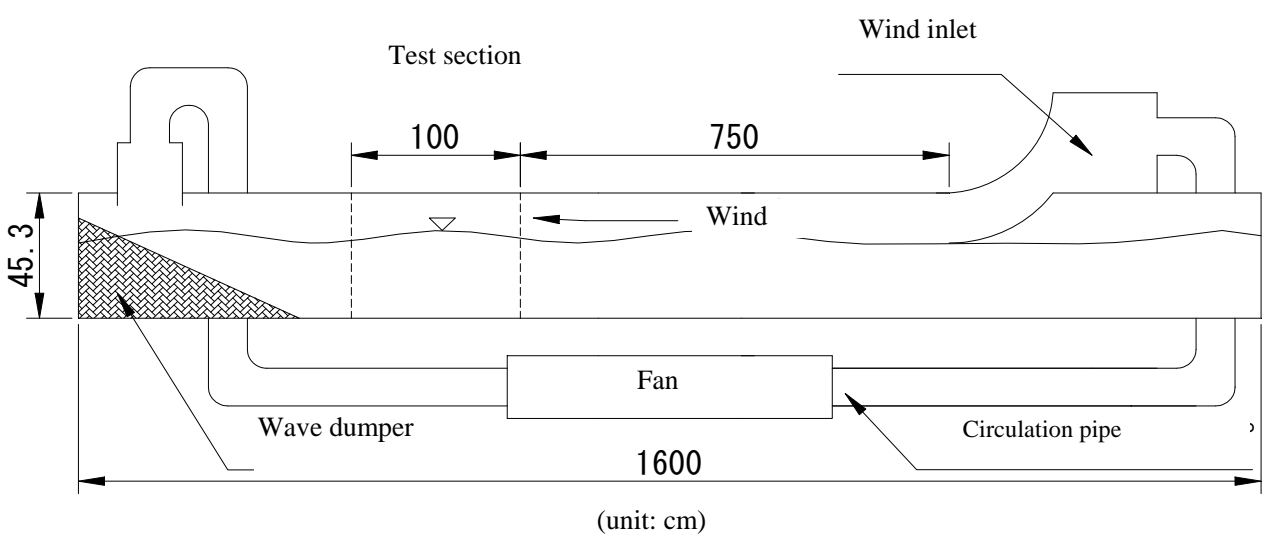

Figure 8. Wind wave tunnel used in this experiment 
schematic diagram of the wind wave tank used in the experiment. Winds were generated by a fan and were introduced into the tank through the inlet. At the end of the tank are an outlet for wind and a wave dumper, which is used to eliminate a reflection wave. The water depth was fixed to $32 \mathrm{~m}$. A test section having a length of $1.0 \mathrm{~m}$ was set at a distance of $7.5 \mathrm{~m}$ from the inlet. The average wind speed was set to $U=14.0 \mathrm{~m} / \mathrm{sec}$.

An 8W YAG CW laser was used as an illumination. A laser beam was expanded two dimensionally using a cylindrical lens. Nylon 12 particles with an average diameter of $80 \mu \mathrm{m}$ and a specific gravity of 1.02 were used as tracer particles. Three high-speed cameras were used for the image capturing. The high-speed video cameras have a pixel count of $1280 \times 1024$ pixels and a frame rate of up to $1000 \mathrm{fps}$. In this experiment, the frame rate was fixed to $125 \mathrm{fps}$, and the exposure time was set to $1 / 250 \mathrm{sec}$. Images captured by the cameras were stored in a computer as 8-bit gray scale images. Five hundred sequential images were captured by each high-speed video camera and were used for the PTV measurements.

Usually, a wind waves has a much longer wave length than its wave height. This means that wide view images are necessary in the velocity measurements. In the experiment, two high-speed video cameras were set side by side horizontally to measure wide areas of wind waves. One high-speed video camera was used to detect the surface of the wind wave. Fluorescent dye was dissolved in water to detect the water surface. When the dissolved fluorescent dye is excited by the laser, the fluorescent dye becomes very bright, whereas the background is very dark. The water surface was detected by using this brightness difference. The three high-speed video cameras used in the experiment were completely synchronized with one another.

\section{Results and discussion}

Fig. 9 shows an example of velocity distributions measured by the PTV. We have successfully obtained velocity vectors near the wind wave surface and measured about eight thousand velocity vectors in the whole measurement area by the PTV. As shown in Fig. 9, the flow structure under the wind wave is complicated because the drift current, the orbital motion and turbulent motion of the wave interact with one another. Near the wind wave surface, the orbital motion is particularly dominant as compared with the drift current and the turbulent motion.

To understand the temporal-spatial properties of vortex motion near the water surface, the vorticity distribution is estimated from the velocity distribution by the proposed MLS method. Fig. 10 shows an example of the time sequence of vorticity distributions. In Fig. 10, black parts indicate the clockwise rotation, and white parts indicate the anticlockwise rotation.

We now focus on a vortex indicated by an arrow in Fig. 10(a). A clockwise vortex motion appears at the water surface behind a wave crest as shown in Fig. 10(a). Then the vortex separates from the water surface and moves downward as shown Fig. 5(b) and Fig. 5(c). Anticlockwise vortices were also observed in other areas. In this research, we have observed generation of a vortex at the water surface, and its separation and downward movement. We have observed that the generation of a vortex at the

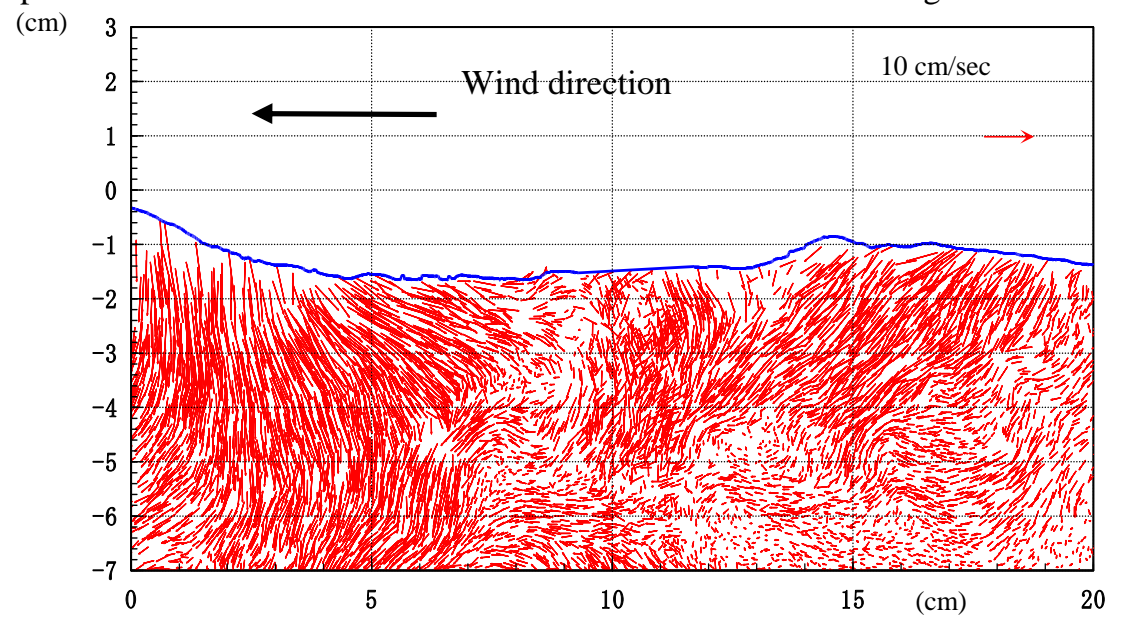

Figure 9. An example of velocity distribution under a wind wave obtained by the PTV 


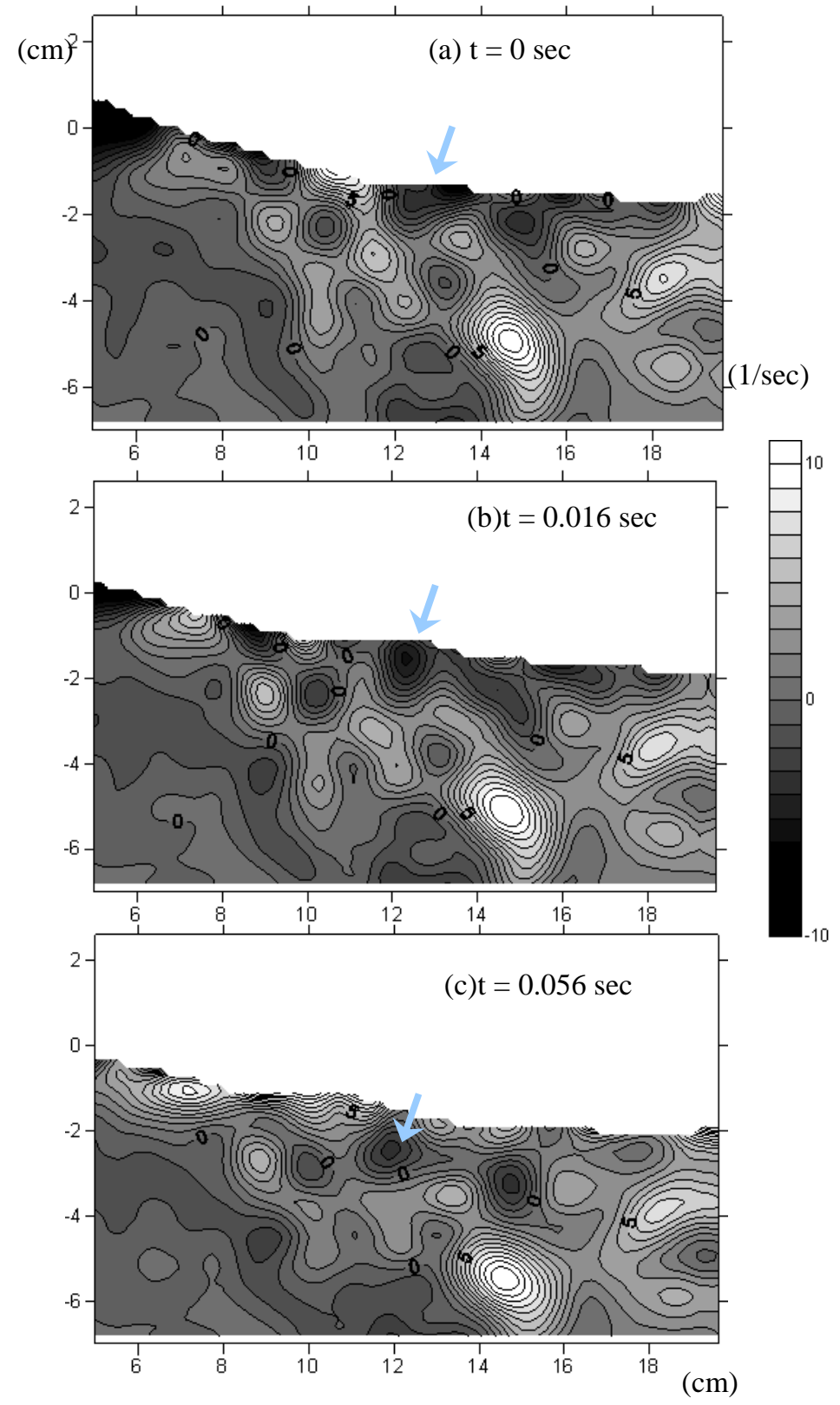

Figure 10. Time sequence of vorticity distributions estimated by MLS method

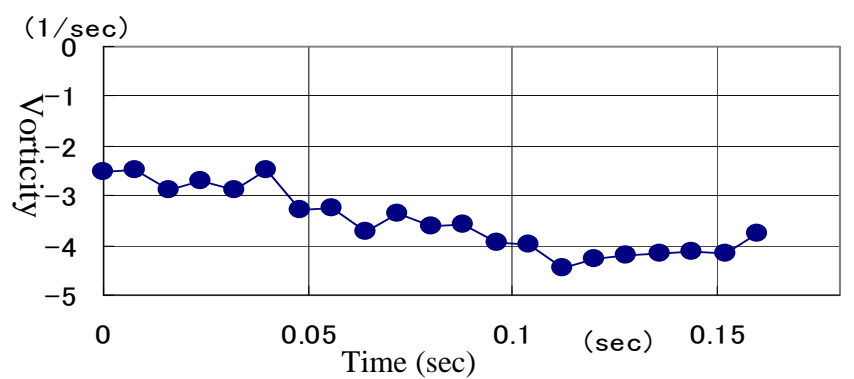

Figure 11. Temporal change of vorticity at the center of the vortex indicated by arrows in Fig. 10

water surface renews the surface water, and the downward movement of the vortex causes the highconcentration $\mathrm{CO}_{2}$ water to transfer to the bulk water.

Fig. 11 shows temporal change of vorticity at the center of the vortex indicated by arrows in Fig.10. The vorticity at the center of a vortex gradually increases with time. It seems that the wind shear acting on the water surface increases the rotation velocity and the scale of the vortex. At a certain rotation 


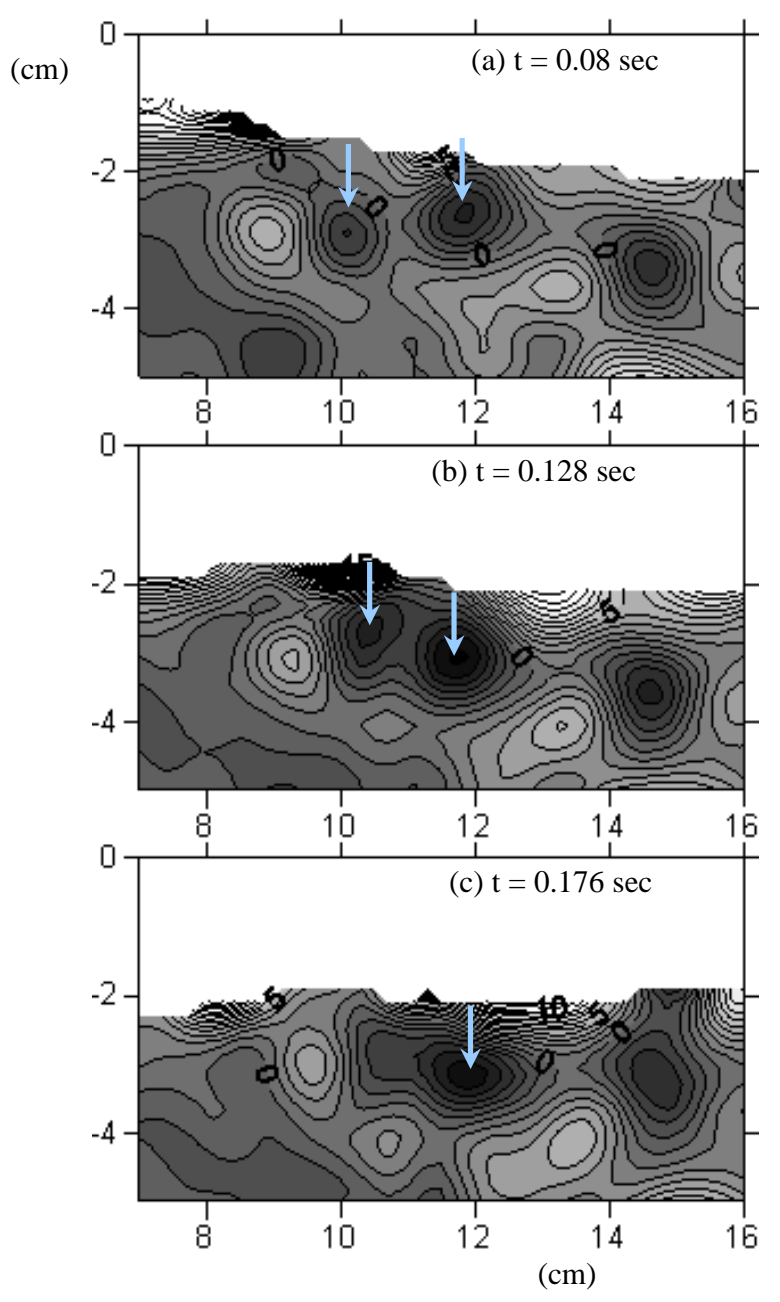

Figure 12. An example of integration of two vortices generated at a water surface

velocity and scale, the vortex separates from the water surface. The vortex then moves downward while the movement of the vortex is interacting with the drift current and the orbital motion of waves. This sequential event of the vortex generation, separation, and movement is repeated irregularly.

As shown in Fig. 12, the integrations of vortices are sometimes observed. Two neighboring vortices indicated by arrows in Fig. 12(a) separate from the water surface, and start to move downward. During the downward movement of the neighboring vortices, the distance between the two vortices becomes shorter as shown in Fig. 12(b). Finally, the two vortices are integrated into one vortex, and the resulting vortex increases its scale.

The generation of a vortex at the water surface by wind wave and the downward movement of the generated vortex are strongly related to the gas transfer at the air-sea interface.

\section{Conclusion}

The vortex generation at the water surface is strongly related to the gas transfer at the water surface. In this study, vortex generation at the water surface under a wind wave was measured using the three high-speed video cameras and using the PTV. The Moving Least Square (MLS) method was proposed to extract vortices accurately from the randomly distributed velocity vectors obtained by the PTV. In this study, the proposed MLS method was applied to measurements of velocity distribution under wind waves. The results obtained in this study are as follows:

1) The MLS method has been proposed to estimate vorticity from the randomly distributed velocities obtained by the PTV. The optimal fitting size of the MLS method has been derived theoretically, and have been confirmed using the Monte Carlo simulation. The accuracy of the proposed MLS method has been compared with that of the commonly used grid conversion method using the simulation data. The results indicate that the relative error of vorticity obtained by the proposed MLS method is always smaller in all cases than that obtained by the gird conversion method. 
2) Flow fields measurements near the wind wave surface have been conducted using the three high-speed video cameras and using the PTV developed by the authors. The proposed MLS method has also been used to estimate vorticity from randomly distributed velocity vectors obtained by the PTV. The results obtained with the PTV indicate that the velocity vectors near the water surface can be measured properly with this method.

3) The time sequence of vorticity distributions obtained by the MLS method shows generation of a vortex at the water surface behind a wave crest. The generated vortex separates from the water surface and moves downward. The vortex moves downward while increasing the rotation speed at the center of the vortex. This observation of vortex generation and downward movement is supported strongly by the downward bursting predicted by Komori et. al. (1993).

4) The integration of two vortices has been observed using the time sequence of vorticity distributions. During downward movement of the vortex generated at the water surface, the vortex is integrated with a neighboring vortex and increases its scale.

\section{ACKNOWLEDGMENTS}

This study is supported by Grant-in-Aid for Scientific Research (B) (20360223) of Japan Society for the Promotion of Science, KAKENHI.

\section{REFERENCES}

Adrian, R.J. (1990): Particle-imaging technique for experimental fluid mechanics, Annual Review of Fluid Mechanics, 23, 261.

Banner, M. L., W.L. Peirson (1998): Tangential stress beneath wind-driven air-water interfaces, $J$. Fluid Mech., Vol. 364, pp.115-145.

Dean, M. A., W.M. Drennan, E.S. Saltzman, R. Wanninkhof, eds (2002): Gas transfer at water surfaces, AGU, 383p.

Devasenathipathy, S., J. G. Santiago, S.T. Wereley, C.D. Meihart, K. Takehara(2003), Particle imaging techniques for microfabricated fluidic systems, Experiments in Fluids, Vol.34, pp. 504-514.

Etoh, T.G., K. Takehara (1995): Development of a new algorithm and supporting technologies for PTV, Proc. of the International Workshop on PIV-Fukui'95, pp.91-106.

Etoh, T., K. Goda, K. Takehara (1999) Evaluation of errors in quadratic approximation and derivation of the optimum size of fitting subdomain in MLS to combine PTV and CFD, Proc. of the $3^{\text {rd }}$ International Workshop PIV '99, Santa Barbara, pp.231-236.

Komori, S., R. Nagaosa and Y. Murakami (1993): Turbulence structure and mass transfer across a sheared air-water interface in wind-driven turbulence, Journal of Fluid Mechanics, Vol. 249, pp.161-183.

Ootsuka, N., K. Takehara, N. Mizutani, G. Tsujimoto, T.G. Etoh and Y. Takano (2003): PTV Measurements of Micro Scale Breaking of Wind Waves, Proceedings of Coastal Engineering, JSCE, Vol.50, pp.86-90 (In Japanese) .

Siddiqui M.H.K., M.R. Loewen, C. Richardson, W.E. Asher and A.T. Jessup (2001): Simultaneous particle image velocimetry and infrared imagery of microscale breaking waves, Physics of Fluids, Vol.13, No. 7, pp.1891-1903.

Takehara, K., R. J. Adrian, G. T. Etoh, and K. T. Christensen (2000): A Kalman tracker for superresolution PIV, Experiments in Fluids, Vol. 29, pp. S34 -S41.

Takehara, K., M. Maki, G. Tsujimoto, T.G. Etoh, Y. Takano(2002): Simultaneous PTV Measurement of Air and Water Flow Close to Water Surfaces under Wind-Wave Conditions, Proceedings of Coastal Engineering, JSCE, Vol.49, pp.66-70 (In Japanese).

Takehara, K., T.G. Etoh, Y. Takano, T. Shigematsu, K. Tanaka (2003): PTV measurements of flow in porous media by using refractivity matching, Proc. of the $7^{\mathrm{TH}}$ Asian Symposium on Visualization, pp.2B2-1-6 .

Takehara, K., Ohtsuka, N., Etoh, T.G., Takano, Y., Tsujimoto, G., Mizutani, N. (2004): Development of Simultaneous plane measurement techniques of air and water flows close to wind wave interfaces, Proc. of the $29^{\text {th }}$ International Conference on Coastal Engineering, pp.1060-1071.

Toba, Y., M. Tokuda, K. Okuda and S. Kawai (1975): Forced convection accompanying wind waves, Journal of Oceanographical Society of Japan, Vol.31, pp192-198.

Yagawa, M. and Y. Sakai (1997): Meshless scheme, Research of Mechanics, Vol. 49, No.1, pp.103110 (In Japanes). 